\title{
Cockayne's syndrome: case report
}

\author{
L. CROME AND G. C. KANJILAL \\ From Queen Mary's Hospital for Children, Carshalton, Surrey, and Cranage Hall Hospital, Holmes Chapel, \\ Cheshire
}

SUMMARY The clinical features in a new non-familial case of Cockayne's syndrome comprised the usual components: dwarfism with microcephaly, severe mental subnormality, progeria-like appearance of the face, pigmented retinopathy, and severe perceptive deafness. The patient also suffered from grand mal epilepsy and died in status epilepticus at the age of 22 years. The neuropathological findings were severe microencephaly, widespread calcifying vasopathy with some secondary degenerative changes in the contiguous tissue, and granular ependymitis.

The course and clinical signs of Cockayne's syndrome are distinctive (Cockayne, 1936, 1946; Neill and Dingwall, 1950; Macdonald, Fitch, and Lewis, 1960; Wilkins, 1965; Moossy, 1967). After initial seemingly normal progress the children begin slowly to regress mentally and physically during the second or third year of life. Microcephaly becomes manifest and they remain dwarfed, having disproportionately long limbs and large hands and feet. The skin of the face is wrinkled, suggesting progeria. It may be pigmented and show scars from solar burns. The eyes are deeply sunken, the nose and chin are often prominent, and the ears large. There is loss of subcutaneous fat in the face. Hearing and sight gradually deteriorate. The eyes show optic atrophy and retinal degeneration of the 'pepper and salt' type. The patients become ataxic and may show a 'tottering' gait, athetoid movements, and coarse intention-type tremor. Ankylosis with flexion deformities and kyphosis may set in and the patients are ultimately bed- or chair-ridden. Radiography may show thickened skull bones and scattered cerebral calcification. Bone age remains within normal limits but there may be evidence of osteoporosis.

All laboratory tests, and these have been numerous in some of the published cases, have yielded no significant information. In a recently described case Fujimoto, Greene, and Seegmiller (1969) observed hyperlipoproteinaemia, hyperinsulinaemia, and renal insufficiency with acidosis.

Although only about a dozen cases have so far been published, at least as recognized instances of the syndrome, a significant proportion were familial (Cockayne, 1936; Neill and Dingwall, 1950; Mac- donald et al., 1960; Paddison, Moossy, Derbes, and Kloepfer, 1963).

The morphological findings have been described fully in only one case recognized as Cockayne's syndrome (Moossy, 1967) and another doubtful one (Beyme, 1946), ${ }^{1}$ but other, indirect, evidence indicates that these changes may be as characteristic as the clinical features. They can be referred to briefly as calcifying vasopathy of the brain. The brains of the two cases reported by Neill and Dingwall were later examined by Dr. R. M. Norman, who indicates that they also showed calcifying vasopathy (Norman and Tingey, 1966). One of us (L.C.) has had an opportunity to examine the histological sections of one of the Australian cases reported by Macdonald et al. (1960). These showed similar calcifying vasopathy but the vascular changes were associated with degeneration of the cerebral cortex indistinguishable from that in Alpers's disease. Lastly, radiography of the head of some of the other patients has confirmed the presence of calcification, at least after a certain age. Neuropathological changes include microencephaly, which may be gross-for example, the brain weighed $460 \mathrm{~g}$ in a woman aged 31 described by Moossy (1967). The main arteries at the base of the brain are normal or show only minimal arteriosclerosis consistent with age. The brain itself may be gritty on cutting, and naked-eye inspection and palpation may reveal calcification in the cerebral white matter, basal ganglia, and

\footnotetext{
${ }^{1}$ A detailed account of another case with full neuropathological findings has been recently published by Ursula Rowlatt in Acta Neuropath. (Berl.), 1969, 14, 52-61.
} 
cerebellum. Histologically, there is marked encrustation of capillaries by colloid hyaline material, some of which is calcified. Some larger vessels show mural impregnation with similar hyaline material and partial or complete calcification. The calcified material frequently gives a positive reaction for iron. The distribution of the vasopathy is characteristic: the heaviest involvement is of the basal ganglia and the cerebral and cerebellar white matter. The cerebral and cerebellar cortices show less severe and less constant focal involvement, while the brain-stem and spinal cord are usually free from calcification. In and around the calcified areas myelin may be lost or show diffuse or patchy pallor on staining. Ependymal granulations may be present upon the ventricular surface.

Other pathological findings are inconstant. The histological changes in the skin of the patients and their relation to those in xerodermic idiocy, Hartnup disease, and porphyria have been discussed by Paddison et al. (1963).

Study of the following exemplar of this, apparently rare, syndrome offered an opportunity to verify earlier accounts of the clinical features and the pathological description of this condition.

\section{CASE REPORT}

The family history of this patient is free from significant mental or neurological disease and two younger siblings are normal. The father was diabetic and an aunt suffered from chorea. The mother was 20 years old at the patient's birth. The pregnancy was normal but the confinement was somewhat prolonged. At birth the infant weighed $2.9 \mathrm{~kg}$.

Development during the first 2 years of life seems to have been normal; the patient sat up by 6 months, walked by 12 , and talked a little by $2 \frac{1}{2}$ years, when she was also clean in her habits but for occasional nocturnal enuresis. However, she did not grow well and at 3 years her speech was still imperfect, so that backwardness was suspected.

At about $3 \frac{1}{2}$ years the mother noticed no reaction when a tray of crockery was accidentally dropped in the child's presence. Thereupon she was found to have impaired hearing and after investigation was placed in a residential school for the deaf. There she was regarded as being mentally backward, although she acquired a modicum of reading and writing. Menarche took place at 13 years but periods were always somewhat irregular. She suffered from light sensitivity and even short exposure to sunlight readily provoked erythema and blistering of the skin so that her open air and seaside outings were discontinued. Retinoscopy showed a 'peculiar granular pigmented appearance of both fundi'. She had gross bilateral perceptive deafness. Her mental level seemed that of a 7 year old child. At the age of 16 she returned home and was extensively investigated at the Manchester Royal Infirmary (Dr. D. Longson).

On examination she was a very small, old-looking girl. The eyes were sunken and vision poor. Breasts were formed and nipples were large. Pubic hair was present. Her height was $115 \mathrm{~cm}$; vertex to pubis $52.5 \mathrm{~cm}$ and pubis to floor $62.5 \mathrm{~cm}$ (Fig. 1).

Investigations included urine ketosteroids and ketogenic steroids; gonadotrophins were low-3 mouse units per day. Radiographs showed normal epiphyseal development for age. No change was seen in the sella or elsewhere in the skull (sic). The urine volume was normal. Fasting blood sugar was $110 \mathrm{mg} / 100 \mathrm{ml}$. Glucose tolerance test was normal. Blood urea was $28 \mathrm{mg} / 100 \mathrm{ml}$. Water diuresis was normal. Urinary amino acids were normal with a suggestion of an excess of cystine. The electrocardiogram was normal. Buccal smears were chromatin positive. The chromosome count was also normal.

After discharge she gradually developed behaviour disturbance associated with delusions of persecution, visual and auditory hallucinations, and she would strike out, screaming and covering her ears. Because of the detrimental effect that this was having on the parents she was admitted at 21 years to Cranage Hall Hospital where she remained till her death. On admission she appeared 
to have greatly reduced vision and hearing. A psychological examination revealed her functioning at a severely subnormal level. None of her previously reported attainments were preserved except for some speech, which was high-pitched and indistinct. Due to her severe sensory deficiencies and inability to write or communicate in any medium, relevant test material was very limited. She was only able to carry out performance items which could be understood by handling alone, and all these were at a very low level. The psychologist (Miss E. A. Hall) observes further that 'by comparison with her other deficiencies Ann's emotions appeared to be remarkably intact. She was able to express tiredness, difficulty, gratitude, annoyance, anger (for example, when I bumped her by mistake), and extreme pleasure when she successfully completed a test item after a prolonged period of trying'.

While in hospital she had frequent temper tantrums and developed for the first time grand mal epilepsy. Her behaviour often suggested a response to hallucinations. An attempt at electroencephalography was unsuccessful because of lack of cooperation, but the record seemed to be within normal limits. Her head circumference was $47 \mathrm{~cm}$. She died in status epilepticus at the age of 22 years.

NECROPSY (Dr. L. Phillips) The body was very small but the shape of the hips, amount of pubic and axillary hair, and external genitalia were normal. The breasts were not well developed though the nipples were bulbous. The skull was very hard and thickened around the circumference, particularly anteriorly. The lungs showed congestion and oedema. Minimal atheroma was present in the aorta and coronary arteries. Other organs and tissues appeared normal.

Histological examination of the somatic tissues showed pulmonary oedema and early bronchopneumonia. Thickening of the intima was present in the aorta and coronary arteries. A small granulomatous lesion was found in the endometrium.

CENTRAL NERVOUS SYSTEM The brain weighed $665 \mathrm{~g}$ with the cerebellum and brain-stem accounting for $55 \mathrm{~g}$. It was symmetrical. The meninges showed generalized slight opacity. The pattern of convolutions was normal. The main arteries at the base of the brain were somewhat rigid but showed only minimal atheroma. The optic nerves and chiasma were small; all other cranial nerves were normal. The brain-stem was reduced in size and indurated. The cerebellum was also considerably smaller than normal, indurated and flattened from above downward (Fig. 2). The whole ventricular system was dilated and the ependyma studded with fine ependymal granulations. The corpus callosum was thin and uneven in thickness. The cerebral white matter showed bluish discolouration in the lateral part of the centrum semiovale. Some parts of the white matter were unduly soft and a gritty sensation was palpable over some areas. The putamen and ventrolateral thalamic nucleus and the white matter of the cerebellum showed similar grittiness and rarefaction. The outlines of the dentate nucleus were blurred but the cerebellar folia showed no naked-eye change.

Blocks from all levels of the central nervous system

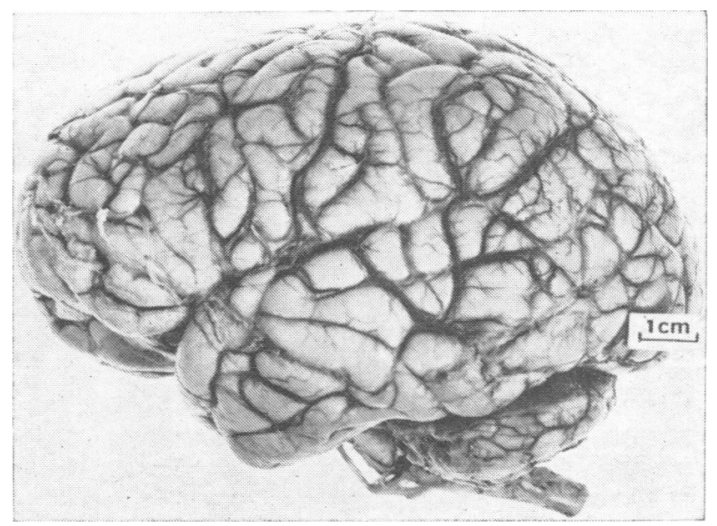

FIG. 2. The brain is small and shows particularly atrophy and flattening of the cerebellum.

were embedded in paraffin and in celloidin. Sections of the celloidin-embedded material were stained with cresyl violet, HVG, by the Holzer method for fibrous glia, and the Heidenhain method for myelin. Sections of the paraffin-embedded material were stained with haematoxylin and eosin, PAS, HVG, and by the von Kossa method for calcium, Perls' method for iron, and the Glees and Marsland method for axis cylinders. Frozen sections were used for fat staining and silver impregnation techniques.

The most striking change was deposition of globules of PAS-positive hyaline material within and upon the outer surface of capillaries. The globules ranged in size from about 1 to $20 \mu$. The smaller ones were discrete while the larger ones, some of which were laminated, tended to coalesce, converting the wall of the vessel into a rough nodular sheath (Fig. 3). Many of the globules and the larger sheaths were fully calcified giving a strongly positive reaction for calcium by the von Kossa method. Some otherwise non-calcified globules presented calcification in their centres. Many of these calcified structures gave also a positive Prussian blue reaction for iron though this was often obscured by counterstain, becoming more obvious when counterstaining was omitted. Some of the capillary lumina were completely obliterated and these, when cut transversely, appeared as extracellular particles of calcium.

Many of the larger vessels, chiefly arterioles, were also abnormal, showing thickening and impregnation of their walls by the hyaline, partly or fully calcified, material. The calcification, if partial, involved the adventitia and outer part of the media, but some vessel walls were, as mentioned, completely calcified. Mural turgidity ranged up to complete vascular occlusion. Some arterioles showed in addition intimal proliferation which, if present, added to the reduction of the lumina.

The above vasopathy was widespread in the cerebral white matter and basal ganglia. It was also present focally in the cerebral cortex affecting all its laminae but particularly the deeper ones. The 'troughs' of the gyri were 

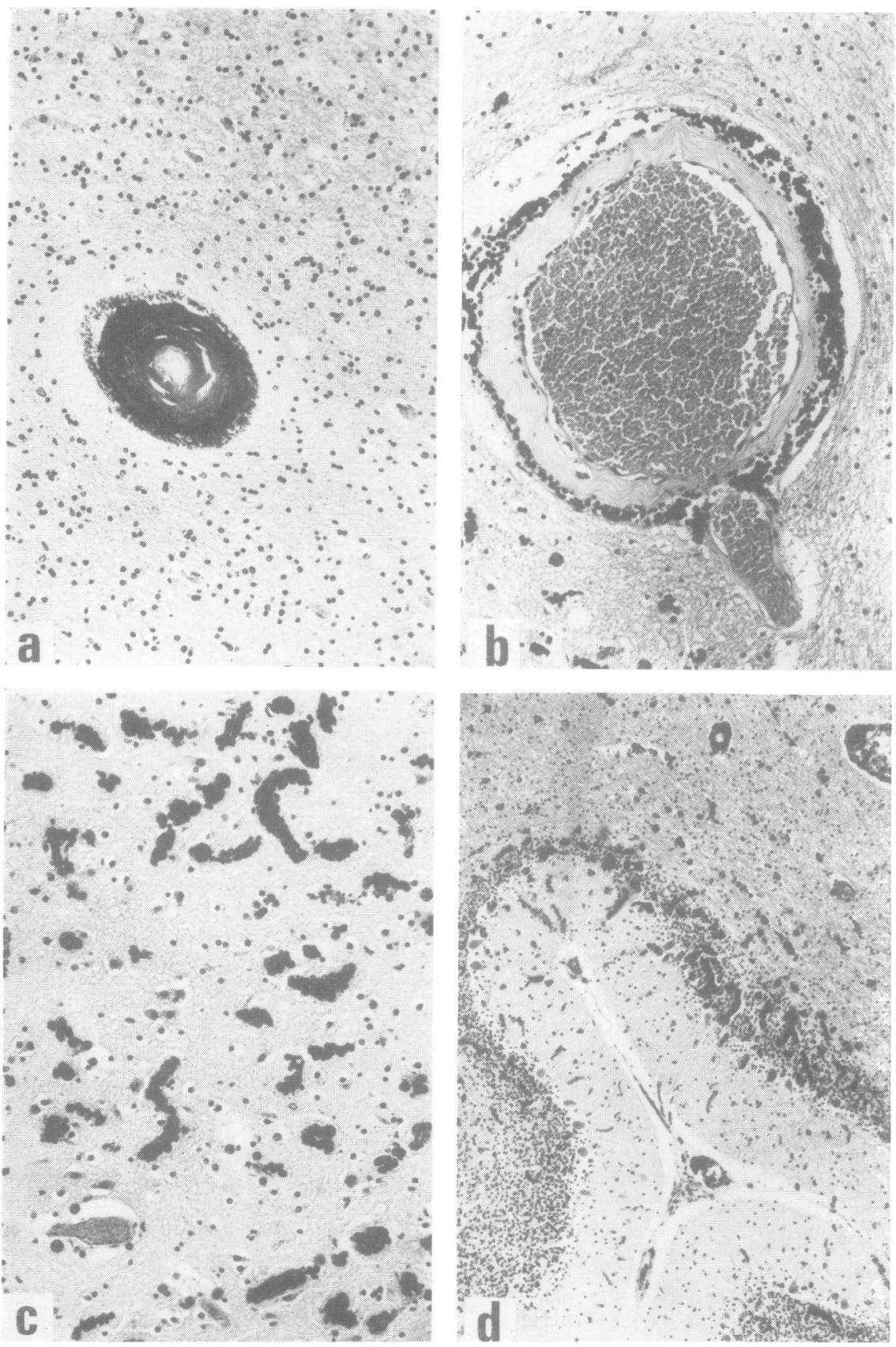

FIG. 3. (a) Almost complete calcification of an arteriole with marked constriction of the lumen, $H$ and $E, \times 160$. (b) A partially calcified vein with hyaline particles in the outer part of the wall, $H$ and $E, \times 160$. (c) Complete calcification of capillaries in the centrum semiovale, $H$ and $E, \times 160$. (d) Calcifying vasopathy of the cerebellum, $H$ and $E, \times 65$. 
generally more often involved than their 'crowns'. Some of these cortical lesions were seen in each of the sections examined but no attempt was made to determine by comprehensive study the relative involvement of different parts of the cerebral cortex. All parts of the cerebral white matter showed more vasopathy than the cortex, while the basal ganglia, except the dorsomedial nucleus of the thalamus, which was relatively spared, showed the greatest involvement.

A few of the abnormal blood vessels were surrounded by hyperplastic and enlarged microglial cells. In most areas affected by the vasopathy structural integrity of the tissue was retained, but in some parts of the white matter and deeper cortical laminae there were also small foci of recent softening surrounded peripherally by a marginal zone of microglial overgrowth (Fig. 4). The more severely affected areas of the white matter and basal ganglia showed, moreover, rarefaction or complete breakdown of the tissue. Preparations stained by the Holzer method displayed diffuse fibrous gliosis of the white matter and marked periventricular gliosis. Marked and dense ependymal granulations were present throughout the ventricular system. The main blood vessels at the base

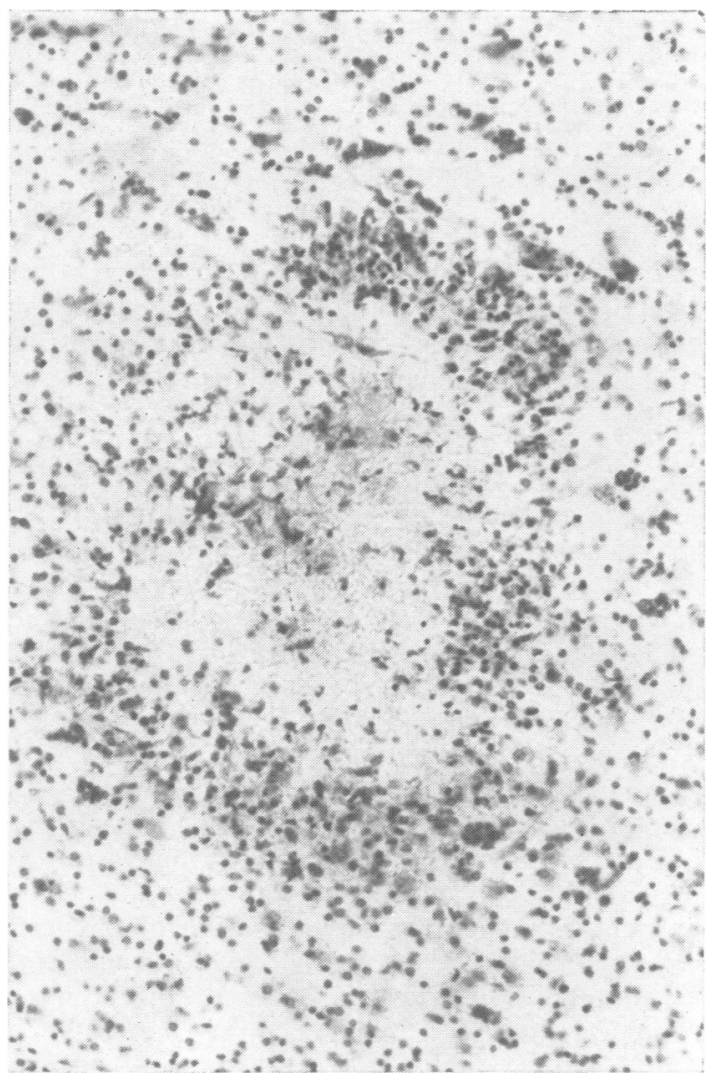

FIG. 4. An area of softening surrounded by a zone of glial overgrowth. $H$ and $E, \times 160$. of the brain and subarachnoid space showed only minimal and doubtful thickening but a few presented proliferation of the intima. The soft meninges contained a slight excess of collagen.

Cortical areas affected by the vasopathy showed some neuronal loss with occasional astrocytic overgrowth. There was similar neuronal depletion in the affected parts of the basal ganglia. Senile plaques and Alzheimer neurofibrillary tangles were searched for but not found.

All the white matter of the cerebral hemispheres showed uneven staining and pallor of myelin, while areas severely involved in the vasopathy were virtually devoid of myelin and of axis cylinders. Myelin was better preserved in the internal capsule and the external sagittal stratum of the occipital lobe (Fig. 5).

The cerebellum presented the same kind of vasopathy as the cerebrum, its white matter being more heavily involved than the cortex (Fig. 6). Its molecular cortical layer was almost entirely unaffected. There was also considerable atrophy of the granular layer and focal loss of Purkinje cells.

The brain-stem and spinal cord showed almost no vasopathy, but scattered throughout these formations

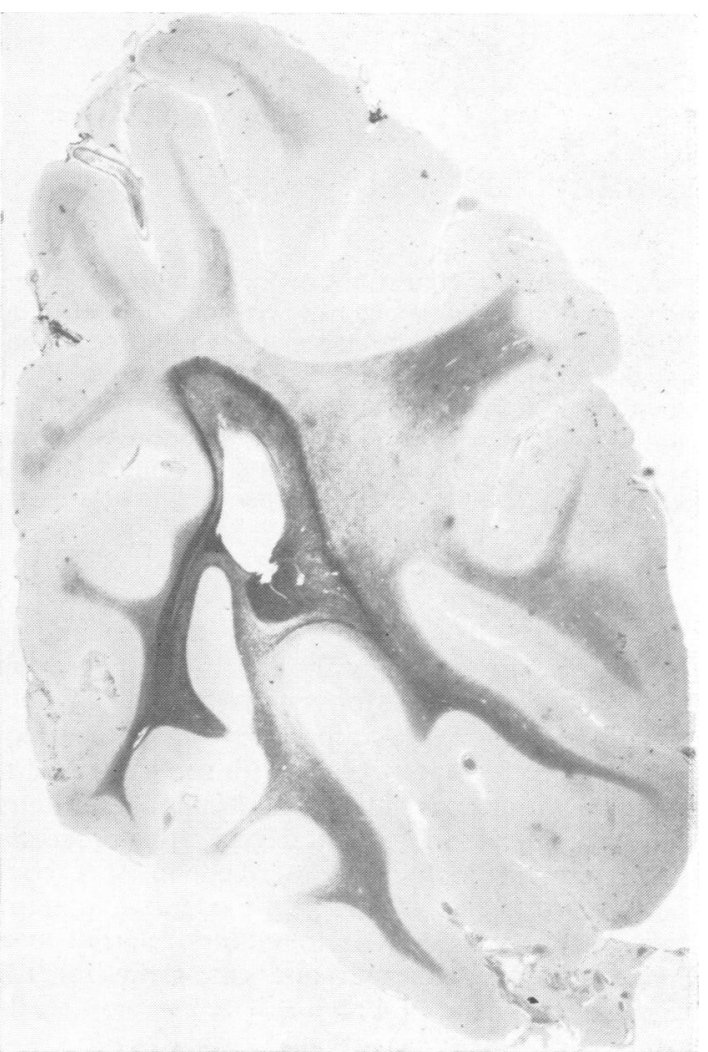

FIG. 5. The occipital lobe showing generalized pallor of myelin staining with preservation of the external sagittal stratum. Heidenhain, $\times 2 \frac{1}{2}$. 


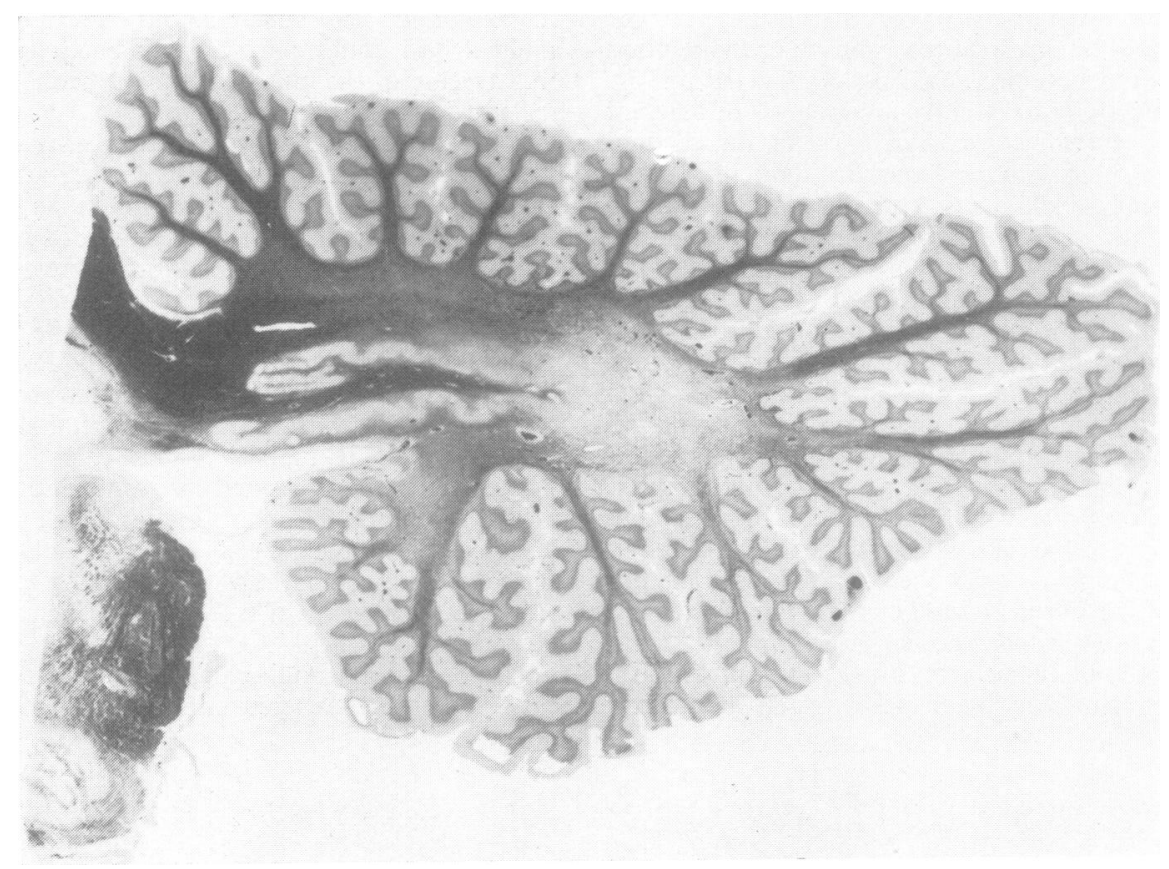

FIG. 6. Cerebellum showing myelin loss in the white matter. Heidenhain, $\times 3$.

were small foci of glial overgrowth, while the zona reticularis of the substantia nigra showed considerable diffuse fibrous gliosis. The white matter of the brain-stem and spinal cord showed pallor of myelin staining which was accentuated focally in many areas. The pyramids and lateral columns of the spinal cord were almost wholly devoid of myelin. The medial and lateral leminisci showed better myelin staining than the other long tracts.

\section{DISCUSSION}

TERMINOLOGY The pathological changes in the present case are strikingly similar to those described by Beyme (1946) and Moossy (1967).

Calcification of the brain is exceedingly common and may occur in association with or as a sequel to such conditions as infection, trauma, tumours, vascular malformations, and degenerative processes. Calcifying vasopathy, as described in this communication, is one of the well-recognized patterns of such calcification. It is sometimes referred to as Fahr's disease. Although one can claim for this eponym the virtue of brevity, it refers only to the pathological state of calcifying vasopathy and not to any defined disease entity. Fahr's single case was a man aged 55 who died rather suddenly and whose preceding history remains quite unknown. Erbslöh and Bochnik (1958), who provide an excellent an beautifully illustrated account of calcifying vas?. pathy, refer to it as 'symmetrische Pseudokalk und Kalkablagerungen im Gehirn'-that is, symmetrical deposits of pseudocalcium or calcium in the brainor "sogenannte idiopathische nichtarteriosklerotische intracerebrale Gefässverkalkungen (Fahr)' - that is, $\mathbb{D}$ so-called idiopathic non-arteriosclerotic intracerebral calcification of blood vessels (Fahr, 1930). They publish a table of conditions with which this pathological state has been associated. These include Addison's disease, diabetes mellitus, progressive muscular dystrophy, hypoparathyroidism, hypothyroidism, hypopituitarism, diseases of the spinal cord, mental retardation, epilepsy, and extrapyramidal syndrome. Cockayne's syndrome is not mentioned among them. It is thus evident that, however distinct the changes of calcifying vasopathy, they are quite unspecific. Moreover, it should also 을 be mentioned that minor degrees of or topologically $D$ restricted calcifying vasopathy may be an incidental asymptomatic pathological finding in the brains of $\mathrm{N}$ people dying from various causes without any evidence of nervous or mental disease. In Cockayne's syndrome the vasopathy is associated with micren- $\omega$ cephaly but the latter is, in its turn, the commonest? 
finding in the brains of the mentally retarded and the coupling of the two anomalies is therefore insufficient for a diagnosis to be made of the syndrome in the absence of the remaining signs-that is, progeria, dwarfism, light sensitivity, and the characteristic eye changes.

VARIABILITY OF THE SYNDROME As with all syndromes formed by multiple components some variability in the manifestation of the Cockayne syndrome must be expected and accepted. Thus, for example, Cockayne's original patients but not the others developed cataracts. Those of Macdonald et al. also showed radiologically an unusual ivorydense condition of the epiphyses of their terminal phalanges. As already mentioned, one of their cases showed an unusual form of cerebral cortical degeneration besides the calcifying vasopathy. Both cases of Neill and Dingwall showed enlargement of the liver, with splenomegaly in one of them. Early greying of the hair was a feature of the case mentioned by Wilkins (1965). The cases of Paddison et al. (1963) and of Cockayne (1946) showed no signs of infantilism.

The relation of some other cases of micrencephaly and calcifying vasopathy to Cockayne's syndrome is more tenuous and it seems debatable whether it is useful to regard them as atypical instances of the syndrome. This applies, for example, to the case reporte 1 by Norman and Tingey (1966). Their patient was microcephalic, progeric, and paretic and suffered also from tremor, epilepsy, contractures, and infantilism. Unlike Cockayne's cases, his fundi were normal and the hearing unimpaired. Pathologically calcifying vasopathy was mild and confined to parts of the basal ganglia. The white matter showed patchy unevenness of myelin staining and loss of myelin which the authors interpreted as leucodystrophy. The two cases described by Jervis (1954) were microcephalic and showed calcifying vasopathy with patchy loss of myelin. Case 1 was, however, severely retarded from birth and showed optic atrophy without retinal changes, and no progeria. Case 2 also showed no retinal changes and no progeria. Equally debatable is the classification of the four cases, two of whom were siblings, described by Hallervorden (1950), and that of HoranyiHechst and Meyer (1939), since they showed neither 'progeria' nor the typical retinal changes.

AETIOLOGY The relative frequency of familial cases in publications on Cockayne's syndrome suggests a recessive mode of inheritance and the operation of some inherited metabolic defect. However, every effort to identify a consistent biochemical lesion has hitherto failed (cf. Fujimoto et al., 1969).
Like the present one, a few of the previously published cases of calcifying vasopathy have shown ventricular ependymal granulations. This, like the meningeal fibrosis, which was also present in these cases, often serves as evidence of foregoing infection -that is, meningitis, ventriculitis, or encephalitis. It is quite true that such granulations are often present after infective conditions, but they are also occasionally encountered in cases without recognized preceding infection. Their pathogenesis is thus still debatable, but infection as a cause of at least some cases of Cockayne's syndrome, although improbable, cannot be definitely ruled out.

The presence of neuropathological signs of ageing in the brain, other than the vasopathy-that is, excessive collagen in the meninges and excess of lipofuscin in many neurones-has been reported by Moossy (1967). Some minimal excess of collagen formation in the meninges was also observed in the present case but there was no excess of neuronal lipofuscin. Senile plaques and neurofibrillary tangles -other changes associated with ageing-were also absent in the present case.

In a recently studied case, Dr. V. Dubowitz (verbal communication) found segmental demyelination in a biopsy of a peripheral nerve. On the basis of this finding and some previously published statements by earlier authors he suggested that Cockayne's syndrome may be a form of leucodystrophy. There was no evidence of leucodystrophy, however, in either of the two cases examined in our laboratory or in the previously published descriptions by Beyme (1946) or Moossy (1967). The case reported by Norman and Tingey (1966) as one of leucodystrophy was not, for reasons already mentioned, quite typical for Cockayne's syndrome.

We are greatly indebted to Dr. L. D. Longson and Dr. L. Phillips whose case notes were used in preparing this report.

\section{REFERENCES}

Beyme, F. (1946). Über das Gehirn einer familiär Oligophrenen mit symmetrischen Kalkablagerungen, besonders in den Stammganglien. Schweiz. Arch. Neurol. Psychiat, 56, 161-190.

Cockayne, E. A. (1936). Dwarfism with retinal atrophy and deafness. Arch. Dis. Childh., 11, 1-8.

Cockayne, E. A. (1946). Dwarfism with retinal atrophy and deafness. Arch. Dis. Childh., 21, 52-54.

Erbslöh, F., and Bochnik, H. (1958). Symmetrische Pseudokalk- und Kalkablagerungen im Gehirn. In Handbuch der speziellen pathologischen Anatomie und Histologie, Vol. 13, edited by W. Scholz. Part 2B. Pp. 1769-1809. Springer: Berlin.

Fahr, Th. (1930). Idiopathische Verkalkung der Hirngefässe. Zbl. allg. Path. path. Anat., 50, 129-133.

Fujimoto, W. Y., Greene, M. L., and Seegmiller, J. E. (1969). Cockayne's syndrome: Report of a case with hyperlipo- 
proteinemia, hyperinsulinemia, renal disease, and normal growth hormone. J. Pediat., 75, 881-884.

Hallervorden, I. (1950). Úber diffuse symmetrische Kalkablagerungen bei einem Krankheitsbild mit Mikrocephalie und Meningoencephalitis. Arch. Psychiat. Nervenkr., 184, 579-600.

Horanyi-Hechst, B., and Meyer, A. (1939). Diffuse sclerosis with preserved myelin islands: a pathological report of a case, with a note on cerebral involvement in Raynaud's disease. J. ment. Sci., 85, 22-28.

Jervis, G. A. (1954). Microcephaly with extensive calcium deposits and demyelination. J. Neuropath. exp. Neurol., 13, 318-329.

Macdonald, W. B., Fitch, K. D., and Lewis, I. C. (1960). Cockayne's syndrome. An heredo-familial disorder of growth and development. Pediatrics, 25, 997-1007.
Moossy, J. (1967). The neuropathology of Cockayne's syndrome. J. Neuropath. exp. Neurol., 26, 654-660.

Neill, C. A., and Dingwall, M. M. (1950). A syndrome resembling progeria: A review of two cases. Arch. Dis. \& Childh., 25, 213-223.

Norman, R. M., and Tingey, A. H. (1966). Syndrome of micrencephaly, striocerebellar calcifications, and leucoes dystrophy. J. Neurol. Neurosurg. Psychiat., 29, 157-163.

Paddison, R. M., Moossy, J., Derbes, V. J., and Kloepfer, W (1963). Cockayne's syndrome. A report of five new cases with biochemical, chromosomal, dermatologic, genetic. and neuropathologic observations. Derm. trop., 2, 195-203

Wilkins, L. (1965). The Diagnosis and Treatment of Endocrine? Disorders in Childhood and Adolescence (Third Edition)? Pp. 168 and 192. Thomas: Springfield, Illinois. 